\title{
ESTUDIO DE LA FLORA LIQUÉNICA DE LAS TURBERAS DE LA COMUNA DE TORTEL (REGIÓN DE AISÉN), PATAGONIA CHILENA.
}

\author{
A STUDY OF THE LICHEN FLORA OF BOGS IN THE COMMUNE \\ OF TORTEL (AISEN REGION), CHILEAN PATAGONIA.
}

Johana Villagra ${ }^{1}$, Drina Montenegro², Cristina San Martín, Carlos Ramírez ${ }^{1}$ \& Iván Álvarez

\begin{abstract}
RESUMEN
Se estudió la flora liquénica de 4 turberas (esfagnosa, pulvinada, pulvinada con Donatia fascicularis y pulvinada con Astelia pumila) en la comuna de Tortel, Patagonia chilena. Se encontraron 40 especies de líquenes, 30 corticícolas y 10 terrícolas, los primeros crecen sobre forófitos leñosos que abundan en la turbera esfagnosa. El espectro de origen es muy amplio sin dominancia de ninguna procedencia. De las 40 especies, 35 crecen en la turbera esfagnosa, 23 en la pulvinada, 6 en la de Donatia fascicularis y 4 en la de Astelia pumila. La similitud florística liquénica ordena las turberas de acuerdo con su origen y con la afinidad florística entre plantas vasculares.
\end{abstract}

Palabras clave: líquenes, biodiversidad, turberas, Patagonia

\section{ABSTRACT}

The lichen flora of 4 bogs (a Sphagnum moss bog; a cushion-forming bog; a cushion bog with Donatia fascicularis; a cushion bog with Astelia pumila) in the Tortel Commune, Chilean Patagonia were studied. Forty species of lichens were found - 30 epiphytic corticolous and 10 terrestrial. The epiphytic ones were growing on trunks of woody phorophytes common on the Sphagnum bog. The origin spectrum is very wide with no clear dominants. Of the 40 species, 35 were found in the Sphagnum bog, 23 in the cushion bog, 6 in the cushion bog plus Donatia fascicularis and 4 in the cushion bog with Astelia pumila. The floristic similarity between lichens on these peatlands can be classified according to their origin and floristic affinities with the associated mosses and vascular plants.

Key words: Lichens, biodiversity, bogs, Chilean Patagonia

1 Instituto de Botánica, Universidad Austral de Chile, Casilla 567, Valdivia. jovyvillagra@gmail.com

2 Ilustre Municipalidad de Tortel, Caleta Tortel.

Proyecto FIA-PI-2004 


\section{INTRODUCCIÓN}

Las turberas son formaciones vegetales que corresponden a pantanos de climas fríos, con una napa freática muy alta y dificultades en el drenaje (Willmanns 1989). La cubierta vegetal de las turberas es continua y presentan una cantidad variables de especies leñosas que sobresalen aisladamente de la cubierta herbácea. Ellas carecen de suelo mineral y el sustrato muy ácido, está formado por materia orgánica (turba) resultante de la lenta e incompleta degradación de la necromasa de las hierbas que la componen, esto es causado por el anegamiento (Ramírez et al. 2002) y también por las temperaturas bajas (Frey \& Lösch 1998, Teneb \& Dollenz 2004, Valenzuela \& Schlatter 2004). Se distinguen turberas topogénicas (turberas bajas) y turberas ombrogénicas (turberas altas), las primeras reciben aporte de agua edáfica con nutrientes y las segundas, dependen exclusivamente del agua de lluvia y por ello, presentan deficiencia de nutrientes minerales, especialmente nitrógeno (Kuntze et al. 1994), esta características se reflejan en la presencia de plantas insectívoras, tales como: Pinguicula antarctica Vahl. (Violeta del pantano) y Drosera uniflora Willd. (Rocío de sol).

En la comuna de Tortel existen turberas topogénicas y también ombrogénicas, las primeras se denominan localmente "mallines colorados" y están dominadas por el musgo Sphagnum magellanicum Brid. (Pon-Pon), las segundas, se llaman "campañas" y están dominadas por plantas vasculares: coníferas leñosas, monocotiledóneas en champa y dicotiledóneas pulvinadas (San Martín et al. 2004). Las primeras se forman en valles de ríos de tierras bajas, mientras que las segundas se desarrollan en altura, por sobre los $400 \mathrm{~m}$, normalmente en lugares rocosos con pendiente. Las turberas esfagnosas (mallines colorados) generalmente tienen un origen secundario, i.e. colonizan lugares donde el primitivo bosques de Nothofagus antarctica (G. Forst.) Oerst. o de Pilgerodendron uvifera (D. Don) Florin ha sido destruido. Las turberas pulvinadas (campañas) son comunidades primarias, i.e. se originaron allí y sobre la roca desnuda. Por lo anterior, presentan una rica flora de plantas vasculares tales como: $L e$ pidothamnus fonckii Phil. (Ciprés enano), Schoenus andinus (Phil.) Pfeiff. (Quilmén) y Embothrium coccineum J.R. Forst et G. Forst.) (Notro). En ella se pueden distinguir variantes con dominancia de plantas herbáceas pulvinadas como Donatia fascicularis J.R. Forst. Et G. Forst. o Astelia pumila (G. Forst.) Gaudich. (Ramírez 1968, San Martín et al. 1999). Sin embargo, la flora liquénica de ellas aún se desconoce en Chile.

El presente estudio trata de averiguar la similitud de las floras liquénicas presentes en los diferentes tipos de turberas mencionadas. Se supone que ellas deberían presentar alta afinidad, ya que presentan características similares tales como frío, cubierta vegetal continua y alta exposición a la luz.

\section{MATERIAL Y MÉTODOS}

\section{Área de estudio}

La comuna de Tortel corresponde a un territorio montañoso muy accidentado, surcado por grandes sistemas fluviales, que desembocan en fiordos y canales. La densidad poblacional es muy baja y su principal característica es el aislamiento. Centros urbanos importantes son Caleta Tortel y Villa O'Higgins.

El clima es marítimo del tipo templado húmedo según Köppen (Scherhag 1969), Amigo \& Ramírez (1998) lo definen como bioclima supratemperado y ultraperhúmedo. Las estaciones meteorológicas más cercanas corresponden a San Pedro ( $47^{\circ} 43^{\prime} \mathrm{S}$; $74^{\circ} 55^{\prime} \mathrm{W}$ ) en la confluencia de los canales Baker y Messier, en el golfo de Penas y la estación de Puerto Edén ( $49^{\circ} 08^{\prime} \mathrm{S} ; 74^{\circ} 25^{\prime} \mathrm{W}$ ), ubicada más al sur en el canal Messier. Los diagramas climáticos de ambas estaciones señalan un exceso de precipitación, con todos los meses del año húmedos (Hajek \& Di Castri 1975). Las diferencias térmicas entre ambas estaciones son pequeñas $\left(8,8^{\circ} \mathrm{C}\right.$ promedio en San Pedro y $7,1^{\circ} \mathrm{C}$ en Puerto Edén). Sin embargo, las diferencias en la precipitación son mayores, sobre $1000 \mathrm{~mm}$ a favor de San Pedro, lo que indica presencia de una gran influencia oceánica (Di Castri \& Hajek 1976), que seguramente llega también a Tortel, ya que Novoa \& Villaseca (1989) lo caracterizan como "agroclima húmedo del río Baker".

La vegetación original está conformada por bosques de coihue de Chiloé (Luzuriago-Nothofagetum nitidae Amigo et al. 2004) en tierras bajas y turberas esfagnosas en los valles fluviales (Pisano 1981, 1983). En altura dominan bosques de coihue 
de Magallanes (Nothofagetum betuloidis Oberdorfer 1960) en quebradas abrigadas y turberas pulvinadas, en lugares expuestos. Bosques de ciprés de las Guaitecas (Pilgerodendronetum uviferae Oberdorfer 1960) colonizan zonas ecotonales en todo el mosaico vegetacional de la comuna.

\section{Métodos}

Entre los años 2004 y 2007 se realizaron prospecciones a los distintos tipos de turbera presentes en la comuna de Tortel, en el marco del Proyecto FIA-PI-C-2004 que realizó una "Evaluación cuantitativa y cualitativa del musgo Sphagnum sp. como forma de conocer su uso, manejo y protección en la comuna de Tortel, región de Aisén". En ellas se colectaron líquenes que fueron preservados siguiendo el modelo propuesto por Lamb (1958). La flora liquénica fue estudiada sistemáticamente en el Instituto de Botánica de la Universidad Austral de Chile y la determinación de especies se hizo en base a literatura específica de Lamb (1958), Galloway (1985, 1992a, 1994), Stenross (1995), Wedin (1995), McCune \& Geiser (1997), Bjerke et al. (2003), Bjerke (2005). Además se recurrió a los reactivos $\mathrm{KOH}$ y en algunos casos $\mathrm{NaCl}$. Posteriormente, se compararon con ejemplares que forman parte de colecciones del Herbario del Museo Nacional de Historia Natural (SGO). El origen fitogeográfico fue obtenido en base a Galloway (1992a, 1994, 1996) y Wedin (1995).

Para comparar las flora liquénica de las turberas, se calculó el índice de similitud de Jaccard
(Sáiz 1980) y se confeccionó una constelación de las comunidades de acuerdo con Ramírez \& Finot (2006), la disimilitud sirvió para establecer la posición de las comunidades turbosas y la similitud para unirlas. Además se determinó el hábitat (sustrato) y el hábito (forma de crecimiento de cada especie).

\section{RESULTADOS Y DISCUSION}

La flora liquénica de las turberas estudiadas está formada por 40 especies (Tabla 1), de ellas un 30\% es endémico de Sudamérica, otro 30\% cosmopolita, el 10\% de los taxa son de origen austral, el 10\% de origen bipolar, $2,5 \%$ de origen neotropical y finalmente, el 2,5\% de taxa con origen en Sudamérica y África. Las especies sin información disponible acerca de su distribución alcanzaron a 15\% (Fig. 1). De acuerdo a estos resultados, el origen de la flora liquénica de turberas, no difiere mayormente del que presenta la flora de líquenes de otras formaciones vegetales. Esta flora liquénica está condicionada por la escasez de un dosel sombreador y por la presencia aislada de pequeños forófitos leñosos, lo que permite la colonización aun cuando no haya espacio desnudo disponible.

De las 40 especies recolectadas, 7 (17,5\%) sólo pudieron ser determinadas a nivel genérico. Las familias con mayor número de especies fueron Parmeliaceae con 14, Lobariaceae con 9 y Cladoniaceae con 5 . Además, hay 3 familias con 2 especies cada una y 6 con sólo una especie. La frecuencia de especies por familias se presenta en la tabla 2. Las familias importantes corresponden a líquenes
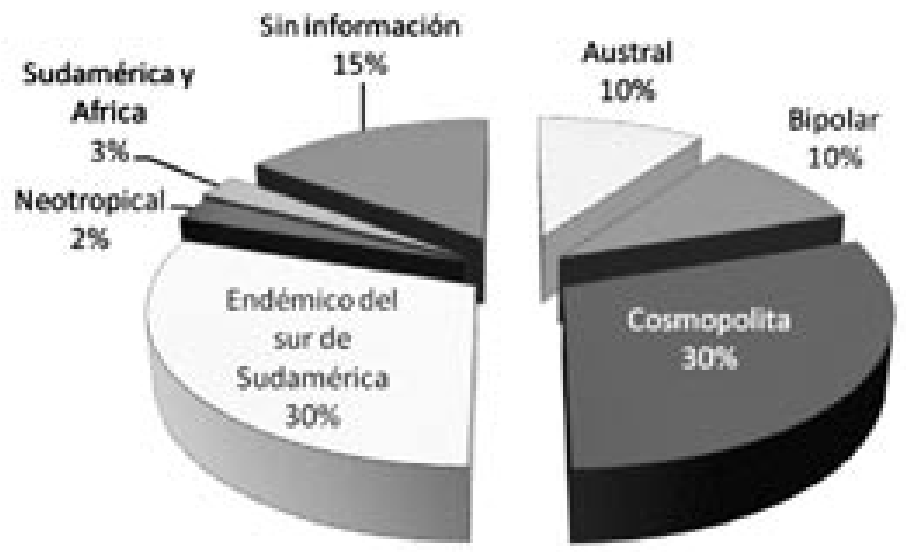

Fig. 1: Origen fitogeográfico de la flora liquénica turbosa estudiada. 
TABLA 1: Flora liquénica de las turberas de la comuna de Tortel (Aisén, Chile).

\begin{tabular}{|c|c|c|c|c|}
\hline Especies liquénicas & Familia & $\mathrm{H}$ & FC & Origen \\
\hline Alectoria nigricans (Ach.) Nyl & Parmeliaceae & $\mathrm{C}$ & $\mathrm{Fr}$ & Bipolar \\
\hline Bryoria chalybeiformis (L.) Brodo \& D. Hawksw. & Parmeliaceae & $\mathrm{C}$ & Fr & Bipolar \\
\hline Cetraria aculeata (Schreb.) FR. & Parmeliaceae & $\mathrm{T}$ & Fr & Cosmopolita \\
\hline Cladina pycnoclada (Pers.) Leight & Cladoniaceae & $\mathrm{T}$ & $\mathrm{C}$ & Endémico Sudamérica \\
\hline Cladonia cariosa (Ach.) Spreng. & Cladoniaceae & $\mathrm{T}$ & $\mathrm{C}$ & Cosmopolita \\
\hline Cladonia chlorophea (Flörke ex Sommerf.) Spreng. & Cladoniaceae & $\mathrm{T}$ & $\mathrm{C}$ & Cosmopolita \\
\hline Cladonia gracilis subsp. elongata (Wulfen) Vain. & Cladoniaceae & $\mathrm{T}$ & $\mathrm{C}$ & Bipolar \\
\hline Cladonia rangiferina (L.) Weber ex F.H. Wigg. & Cladoniaceae & $\mathrm{T}$ & $\mathrm{C}$ & Bipolar \\
\hline Coelopogon cf. epiphorellus (Nyl.) Brusse \& Kärnefelt & Parmeliaceae & $\mathrm{C}$ & Fr & Sudamérica y Africa \\
\hline Degelia gayana (Mont) Arv. \& D. J. Galloway & Pannariaceae & $\mathrm{C}$ & $\mathrm{F}$ & Austral \\
\hline Dictyonema glabratum (Spreng.) D. Hawksw & Thelephoraceae & $\mathrm{C}$ & $\mathrm{F}$ & Endémico Sudamérica \\
\hline Erioderma glauscens (Taylor) Mull & Pannariaceae & $\mathrm{C}$ & $\mathrm{F}$ & Endémico Sudamérica \\
\hline Graphis scripta (L.) Ach. & Graphiceaceae & $\mathrm{C}$ & $\mathrm{Cr}$ & Cosmopolita \\
\hline Hypogymnia sp. & Parmeliaceae & $\mathrm{C}$ & $\mathrm{F}$ & Sin información \\
\hline Hypotrachyna sinuosa (Sm) Hale & Parmeliaceae & $\mathrm{C}$ & $\mathrm{F}$ & Cosmopolita \\
\hline Lecanora sp. & Lecanoraceae & $\mathrm{C}$ & $\mathrm{Cr}$ & Sin información \\
\hline Leifidium tenerum (Laurer) Wedin & Sphaerophoraceae & $\mathrm{C}$ & $\mathrm{Fr}$ & Austral \\
\hline Menegazzia wilsonii (Vain. Ex Räsänen) Bjerke. & Parmeliaceae & $\mathrm{C}$ & $\mathrm{F}$ & Endémico Sudamérica \\
\hline Menegazzia cinccinata (Ach.) Bitter & Parmeliaceae & $\mathrm{C}$ & $\mathrm{F}$ & Endémico Sudamérica \\
\hline Menegazzia globulifera R. Sant.Type & Parmeliaceae & $\mathrm{C}$ & $\mathrm{F}$ & Austral \\
\hline Menegazzia sanguinascens (Räsänen) R. Sant. & Parmeliaceae & $\mathrm{C}$ & $\mathrm{F}$ & Endémico Sudamérica \\
\hline Nephroma antarcticum Müll. Arg. & Nephromataceae & $\mathrm{C}$ & $\mathrm{F}$ & Endémico Sudamérica \\
\hline Nephroma cellulosum (Ach.) Ach. & Nephromataceae & $\mathrm{C}$ & $\mathrm{F}$ & Austral \\
\hline Parmotrema chinense (Osbeck) Hale \& Ahti & Parmeliaceae & $\mathrm{C}$ & $\mathrm{F}$ & Cosmopolita \\
\hline Platismatia glauca (L.) W. Culb. \& C. Culb & Parmeliaceae & $\mathrm{C}$ & $\mathrm{F}$ & Cosmopolita \\
\hline $\begin{array}{l}\text { Pseudocyphellaria berberina (G. Forster) } \\
\text { D. J. Galloway \& P. James }\end{array}$ & Lobariaceae & $\mathrm{C}$ & $\mathrm{F}$ & Endémico Sudamérica \\
\hline Pseudocyphellaria crocata (L.) Vain. & Lobariaceae & $\mathrm{C}$ & $\mathrm{F}$ & Cosmopolita \\
\hline Pseudocyphellaria encoensis R. Sant. & Lobariaceae & $\mathrm{C}$ & $\mathrm{F}$ & Neotropical \\
\hline Pseudocyphellaria freycinetii (Delise) Malme & Lobariaceae & $\mathrm{T}$ & $\mathrm{F}$ & Endémico Sudamérica \\
\hline Pseudocyphellaria intricata (Delise) Vain. & Lobariaceae & $\mathrm{C}$ & $\mathrm{F}$ & Endémico Sudamérica \\
\hline Pseudocyphellaria obvoluta (Ach.) Malme & Lobariaceae & $\mathrm{C}$ & $\mathrm{F}$ & Endémico Sudamérica \\
\hline Pseudocyphellaria sp & Lobariaceae & $\mathrm{C}$ & $\mathrm{F}$ & Sin información \\
\hline Ramalina sp. & Ramalinaceae & $\mathrm{C}$ & $\mathrm{Fr}$ & Sin información \\
\hline Stereocaulon alpinum Laurer & Stereocaulaceae & $\mathrm{T}$ & $\mathrm{Fr}$ & Cosmopolita \\
\hline Stereocaulon ramulosum (Sw.) Räuschel & Stereocaulaceae & $\mathrm{T}$ & Fr & Cosmopolita \\
\hline Sticta fuliginosa (Hoffm.) Ach. & Lobariaceae & $\mathrm{C}$ & $\mathrm{F}$ & Cosmopolita \\
\hline Sticta hypochra Vain. & Lobariaceae & $\mathrm{C}$ & $\mathrm{F}$ & Endémico Sudamérica \\
\hline Thamnolia vermicularis Ach. Ex Schaer & Icmadophilaceae & $\mathrm{T}$ & Fr & Cosmopolita \\
\hline Usnea sp1 & Parmeliaceae & $\mathrm{C}$ & $\mathrm{Fr}$ & Sin información \\
\hline Usnea sp2 & Parmeliaceae & $\mathrm{C}$ & $\mathrm{Fr}$ & Sin información \\
\hline
\end{tabular}

H (Habitat): $\mathrm{C}=$ corticícola, $\mathrm{T}=$ terrícola. FC (Forma de Crecimiento): $\mathrm{C}=$ crustáceo,

$\mathrm{F}=$ folioso, $\mathrm{Fr}=$ fruticuloso.

foliosos y fruticulosos que colonizan principalmente forófitos leñosos. Hay que destacar la presencia de Erioderma glauscens y Pseudocyphellaria obvoluta, especies citadas por primera vez para la región de Aisén, pero citadas para Chile previamente por Pereira (2007) en el Parque Katalapi (Región de
Los Lagos) la primera, y por Galloway (1986) para las regiones de la Araucanía y de Magallanes, la segunda.

Sólo se encontraron líquenes de hábitat terrícola y principalmente corticícolas registrándose 10 y 30 especies, respectivamente (Tabla 3). El 
TABLA 2: Distribución de la flora liquénica de las turberas estudiadas por familia.

\begin{tabular}{|l|c|c|}
\hline Familia & Especies & Porcentaje \\
\hline Parmeliaceae & 14 & 35,0 \\
\hline Lobariaceae & 9 & 22,5 \\
\hline Cladoniaceae & 5 & 12,5 \\
\hline Nephrotomaceae & 2 & 5,0 \\
\hline Pannariaceae & 2 & 5,0 \\
\hline Stereocaulaceae & 2 & 5,0 \\
\hline Sphaerophoraceae & 1 & 2,5 \\
\hline Thelephoraceae & 1 & 2,5 \\
\hline Graphiceaceae & 1 & 2,5 \\
\hline Lecanoraceae & 1 & 2,5 \\
\hline Ramalinaceae & 1 & 2,5 \\
\hline Icmadophilaceae & 1 & 2,5 \\
\hline Total (12 familias) & 40 & 100 \\
\hline
\end{tabular}

hábito o forma de crecimiento más abundante fue el de líquenes foliosos con 22 especies, le siguen en importancia los líquenes fruticulosos con 11 especies, los líquenes crustáceos sólo presentaron 2 especies (Tabla 4 y Fig. 2). Además, hay 5 especies con forma de crecimiento compuesto, es decir, con base escuamulosa y ramificación fruticulosa. La forma de crecimiento foliosa, más abundante, tiene hábito corticícola y crece sobre las plantas leñosas que colonizan las turberas, esto guarda estrecha relación con el clima de la región que presenta un exceso de precipitación y alta humedad ambiental que favorece el desarrollo de epífitos. La ausencia o escasez del hábito crustáceo se debe seguramente a la falta de un sustrato rocoso, por la alta cobertura vegetal de las turberas, que siempre supera el 100\%, debido a la estratificación que originan las plantas leñosas arbóreas y arbustivas.

La mayor cantidad de líquenes se encontró en la turbera esfagnosa con 35 especies, mientras que la turbera pulvinada, alcanzó a 23 especies (Tabla 5). Las turberas pulvinada con Donatia y con Astelia, consideradas aquí como variantes de la turbera pulvinada, presentaron 6 y 4 especies liquénicas, respectivamente. Esta distribución de la riqueza florística liquénica en las distintas turberas estudiadas está directamente relacionada con la cantidad de forófitos disponibles en cada una de ellas. A mayor cantidad de forófitos mayor cantidad de líquenes. Las variantes de Donatia y Astelia prácticamente carecen de forófitos, lo cual está de acuerdo con su escasa flora liquénica y con el crecimiento pulviniforme de las especies dominantes Donatia fascicularis y Astelia pumila, que cubre completamente el sustrato turboso.

En la turbera pulvinada faltaron los elementos neotropicales y de Sudamérica y África, mientras que la turbera esfagnosa presentó todo el amplio espectro de origen fitogeográfico liquénico, lo que se corresponde con la mayor abundancia de hábitats en la turbera esfagnosa. En ambas turberas los elementos dominantes fueron el endémico del sur de América y el cosmopolita, aunque el primero domina en la esfagnosa y el segundo, en la pulvinada.

Cladina pycnoclada (Pers.) Leight., Cladonia rangiferina (L.) F.H. Wigg. y Menegazzia sanguinascens (Resanen) R. Sant. son las únicas especies que se presentaron en las cuatro turberas con un 100\% de frecuencia (Tabla 5). Las dos cladoniáceas tienen forma de crecimiento compuesto y hábitat terrícola, mientas que Menegazzia sanguinascens, es un líquen folioso que coloniza la base de los forófitos. De acuerdo a la anterior, podría considerarse a estas especies como características de las formaciones turbosas abiertas. Las especies, Cladonia gracilis (L.) Willd. subsp. elongata, Cetraria aculeata (Schreb.) Fr. y Usnea sp1 se presentaron en 3 tipos de turberas con

TABLA 3: Número de especies liquénicas por hábitat en las diferentes turberas estudiadas.

\begin{tabular}{|l|c|c|c|c|}
\hline Hábitat/Turbera: & Esfagnosa & Pulvinada & Con Donatia & Con Astelia \\
\hline Corticícola & 30 & 13 & 2 & 1 \\
\hline Terrícola & 5 & 10 & 4 & 3 \\
\hline
\end{tabular}

TABLA 4: Número de especies liquénicas por hábito (forma de crecimiento) distribuidas en las turberas estudiadas.

\begin{tabular}{|l|c|c|c|c|}
\hline Hábito / Turbera: & Esfagnosa & Pulvinada & Con Donatia & Con Astelia \\
\hline Folioso & 21 & 11 & 1 & 1 \\
\hline Fruticuloso & 8 & 6 & 2 & 1 \\
\hline Compuesto & 4 & 5 & 3 & 2 \\
\hline Crustáceo & 2 & 1 & 0 & 0 \\
\hline
\end{tabular}




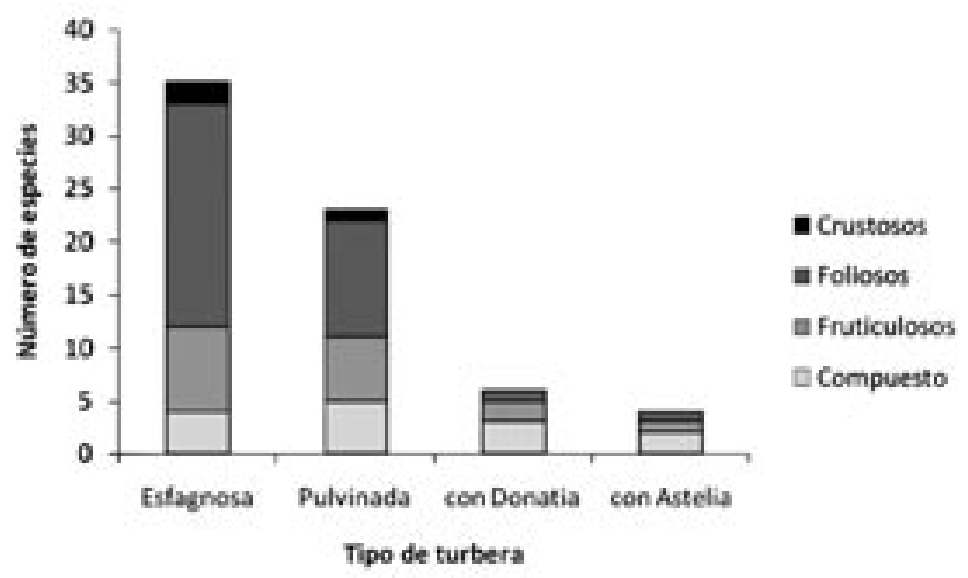

Fig. 2: Distribución de especies por formas de vida (Hábito) en la flora de las turberas estudiadas.

un $75 \%$ de frecuencia. De ellas las dos primeras son terrícolas y la última, corticícola. Cladonia gracilis presenta una forma de crecimiento compuesta y las otras dos especies, una forma fruticulosa, que se adhiere a los cojines de las especies pulvinadas que conforman las turberas. Además hay 13 especies compartidas por dos turberas, la esfagnosa y la pulvinada y 21 especies que sólo están presentes en una sola turbera, 17 de ellas crecen en la turbera esfagnosa y 4 en la pulvinada.

Al someter las 4 turberas a un análisis de similitud florística considerando sólo la presencia/ausencia de las especies liquénicas, la mayor similitud $(66,7 \%)$ se encuentra entre las dos variantes de la turbera pulvinada que, además, presentan menor cantidad de especies (Tabla 6). Una afinidad florística menor al $50 \%$ se presenta entre la turbera esfagnosa y la pulvinada. Los menores valores se encuentran entre estas dos turberas y las variantes con Donatia y con Astelia, que a su vez presentan una mayor similitud florística liquénica con la turbera pulvinada, lo que está de acuerdo con el carácter de variantes propuesto para ambas. También hay que considerar que en estas dos variantes con cojines monoespecíficos, son muy escasos los forófitos leñosos.

La constelación de comunidades turbosas de acuerdo a su similitud florística liquénica concuerda con lo expresado anteriormente, demostrando que hay mayor similitud florística entre las turberas pulvinadas con Donatia y Astelia, que entre aquellas turbosas y pulvinadas (Fig. 3). Además, estas últimas comunidades turbosas están alejadas de

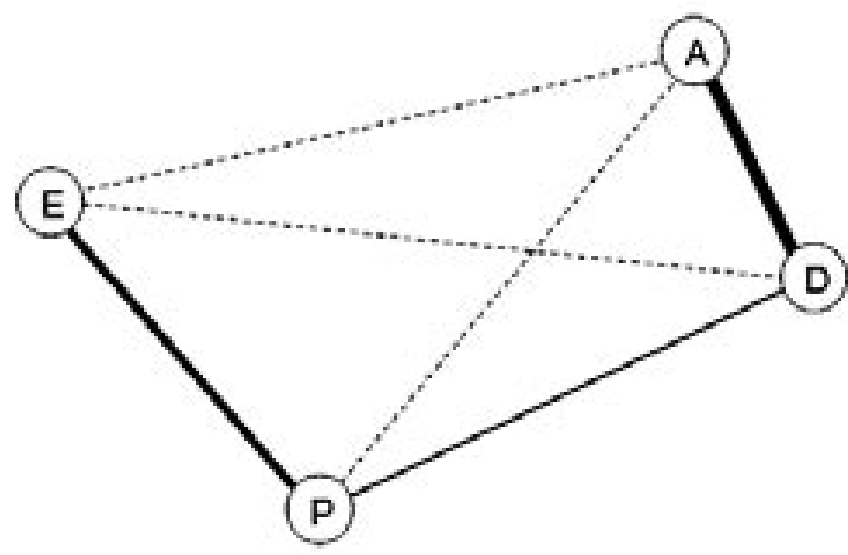

Fig. 3: Constelación de comunidades turbosas estudiadas, según la similitud/disimilitud de su flora liquénica. Turberas: $\mathrm{E}=$ esfagnosa, $\mathrm{P}=$ pulvinada, $\mathrm{D}=$ Con Donatia y $\mathrm{A}$ = Con Astelia. Las líneas de unión más gruesas indican mayor similitud. 
TABLA 5: Presencia de especies liquénicas en las diferentes turberas estudiadas, ordenadas por frecuencia en orden descendente

\begin{tabular}{|c|c|c|c|c|c|}
\hline Especies & Esfagnosa & Pulvinada & Con Donatia & Con Astelia & $\mathrm{F}$ \\
\hline Cladina pycnoclada & $\mathrm{x}$ & $\mathrm{X}$ & $\mathrm{x}$ & $\mathrm{x}$ & 4 \\
\hline Cladonia rangiferina & $\mathrm{x}$ & $\mathrm{X}$ & $\mathrm{x}$ & $\mathrm{x}$ & 4 \\
\hline Menegazzia sanguinascens & $\mathrm{x}$ & $\mathrm{x}$ & $\mathrm{x}$ & $\mathrm{x}$ & 4 \\
\hline Usnea sp1 & $\mathrm{x}$ & $\mathrm{x}$ & $\mathrm{x}$ & & 3 \\
\hline Cladonia gracilis subsp. elongata & $\mathrm{x}$ & $\mathrm{x}$ & $\mathrm{x}$ & & 3 \\
\hline Cetraria aculeata & & $\mathrm{x}$ & $\mathrm{x}$ & $\mathrm{x}$ & 3 \\
\hline Stereocaulon ramulosum & $\mathrm{x}$ & $\mathrm{x}$ & & & 2 \\
\hline Menegazzia cinccinata & $\mathrm{x}$ & $\mathrm{x}$ & & & 2 \\
\hline Usnea sp2 & $\mathrm{x}$ & $\mathrm{x}$ & & & 2 \\
\hline Sticta fuliginosa & $\mathrm{x}$ & $\mathrm{x}$ & & & 2 \\
\hline Sticta hypochra & $\mathrm{x}$ & $\mathrm{x}$ & & & 2 \\
\hline Parmotrema chinense & $\mathrm{x}$ & $\mathrm{x}$ & & & 2 \\
\hline Menegazzia wilsonii & $\mathrm{x}$ & $\mathrm{x}$ & & & 2 \\
\hline Cladonia cariosa & $\mathrm{x}$ & $\mathrm{x}$ & & & 2 \\
\hline Nephroma cellulosum & $\mathrm{x}$ & $\mathrm{x}$ & & & 2 \\
\hline Hypogymnia sp & $\mathrm{x}$ & $\mathrm{x}$ & & & 2 \\
\hline Pseudocyphellaria crocata & $\mathrm{x}$ & $\mathrm{x}$ & & & 2 \\
\hline Graphis scripta & $\mathrm{x}$ & $\mathrm{x}$ & & & 2 \\
\hline Pseudocyphellaria sp & $\mathrm{x}$ & $\mathrm{x}$ & & & 2 \\
\hline Thamnolia vermicularis & & $\mathrm{x}$ & & & 1 \\
\hline Cladonia chlorophea & & $\mathrm{x}$ & & & 1 \\
\hline Stereocaulon alpinum & & $\mathrm{x}$ & & & 1 \\
\hline Pseudocyphellaria freycinetii & & $\mathrm{x}$ & & & 1 \\
\hline Menegazzia globulifera & $\mathrm{x}$ & & & & 1 \\
\hline Bryoria chalybeiformis & $\mathrm{x}$ & & & & 1 \\
\hline Alectoria nigricans & $\mathrm{x}$ & & & & 1 \\
\hline Coelopogon cf. epiphorellus & $\mathrm{x}$ & & & & 1 \\
\hline Leifidium tenerum & $\mathrm{x}$ & & & & 1 \\
\hline Platismatia glauca & $\mathrm{x}$ & & & & 1 \\
\hline Degelia gayana & $\mathrm{x}$ & & & & 1 \\
\hline Erioderma glauscens & $\mathrm{x}$ & & & & 1 \\
\hline Dictyonema glabratum & $\mathrm{x}$ & & & & 1 \\
\hline Nephroma antarcticum & $\mathrm{x}$ & & & & 1 \\
\hline Hypotrachyna sinuosa & $\mathrm{x}$ & & & & 1 \\
\hline Pseudocyphellaria berberina & $\mathrm{x}$ & & & & 1 \\
\hline Pseudocyphellaria encoensis & $\mathrm{x}$ & & & & 1 \\
\hline Pseudocyphellaria intricata & $\mathrm{x}$ & & & & 1 \\
\hline Pseudocyphellaria obvoluta & $\mathrm{x}$ & & & & 1 \\
\hline Ramalina $s p$ & $\mathrm{x}$ & & & & 1 \\
\hline Lecanora $s p$ & $\mathrm{x}$ & & & & 1 \\
\hline Total 40 especies & 35 & 23 & 6 & 4 & \\
\hline
\end{tabular}

aquellas variantes con Donatia y Astelia, aunque la mayor distancia se presenta con la turbera esfagnosa. Nuevamente se confirma la mayor afinidad de las variantes con la turbera pulvinada, a la cual pertenecerían. De acuerdo a lo anterior, la afinidad florística liquénica entre turberas, corresponde a la relación de origen y a la afinidad florística de musgos y plantas vasculares entre ellas.

En las turberas esfagnosas y pulvinadas abundan los líquenes de hábitat corticícola (30 y 13 especies, respectivamente), mientras que formas de hábitat terrícola se reducen a 5 en la primera y a 10 
TABLA 6: Porcentaje de similitud (triángulo superior) y de disimilitud (triángulo inferior) florística liquénica entre las turberas estudiadas. La diagonal de 100\% separa ambos triángulos.

\begin{tabular}{|l|c|c|c|c|}
\hline Turberas & Esfagnosa & Pulvinada & Con Donatia & Con Astelia \\
\hline Esfagnosa & $\mathbf{1 0 0}$ & 45,0 & 13,9 & 8,3 \\
\hline Pulvinada & 55,0 & $\mathbf{1 0 0}$ & 26,1 & 17,4 \\
\hline Con Donatia & 86,1 & 73,9 & $\mathbf{1 0 0}$ & 66,7 \\
\hline Con Astelia & 91,7 & 82,6 & 33,3 & $\mathbf{1 0 0}$ \\
\hline
\end{tabular}

en la segunda. Al expresar las diferencias descritas en porcentaje, se observa que la forma corticícola disminuye su participación en la turbera pulvinada, mientras que la terrícola casi la cuadruplica. La forma de crecimiento folioso domina en la turbera esfagnosa con un $60 \%$ y en la turbera pulvinada sólo presenta un $25 \%$.

Los líquenes se consideran organismos pioneros que colonizan roca desnuda y conforman las primeras etapas en la descomposición de ésta, para formar el suelo y permitir así el desarrollo de las primeras etapas de la sucesión vegetal en terrenos desnudos. Por esta razón necesitan espacio que colonizar, los cuales son escasos en la turbera dada la alta cobertura vegetal, pero los forófitos ofrecen dicho espacio en sus troncos, los que cambian sus condiciones como hábitat a medida que aumenta la altura del tronco (Pharo \& Beattie 2002), cuando tienen o carecen de hojas en el caso de los árboles caducifolios y cuando crecen aislados o en comunidades boscosas. Lo anterior explica la mayor cantidad de especies liquénicas en la turbera esfagnosa, que presenta una mayor cantidad de forófitos leñosos, como por ejemplo: Nothofagus antarctica (Nire), Nothofagus betuloides (Mirb.) Oerst. (Coihue de Magallanes) y Pilgerodendron uvifera (Ciprés de las Guaitecas). Nuestros resultados coinciden con los de Galloway (1992b) para el Parque Nacional "Laguna San Rafael" porque existe dominancia de epífitos liquénicos foliosos (por ejemplo Pseudocyphelaria sp. y Sticta sp.) sólo en presencia de forófitos, mientras que en lugares más expuestos (dunas, marismas) abundan las especies de Cladonia y Stereocaulon. La presencia de forófitos favorece la retención de la humedad atmosférica, lo que permite la proliferación de los líquenes foliosos (Redón et al. 1979, Pereira \& San Martín 1998, Pereira et al. 1999).

Como organismos mixtos de ficobionte y micobionte (Grube \& Hawksworth 2007), los líquenes soportan condiciones desfavorables y extremas en la naturaleza como sequía, temperatura y lumino- sidad, pero no son resistentes a la contaminación atmosférica de origen antrópico (Galloway 1992b, Brunialti \& Giordani 2003, Calvelo \& Liberatore 2004). Además, por su carácter de organismos poiquilohídricos han conseguido alcanzar óptimos fotosintéticos en bajas condiciones de hidratación (Larcher 1994). Por lo anterior, su presencia con alto número de especies en las comunidades turbosas, indica condiciones atmosféricas de pristinidad, como sucede en la comuna de Tortel. La riqueza de especies liquénicas en estepas de altura y en bosques de Nothofagus de la Patagonia chilena no ha sido estudiada y se mantiene como un campo inexplorado.

\section{AGRADECIMIENTOS}

Los autores agradecen el financiamiento del Fondo de Investigación Agraria a través del Proyecto FIA-PI-2004 y el cofinanciamiento de la Ilustre Municipalidad de Tortel y a un revisor anónimo que nos aportó valiosas sugerencias.

\section{LITERATURA CITADA}

Amigo, J. \& C. Ramírez 1998. A bioclimatic classification of Chile: woodland communities in the temperate zone. Plant Ecology 136: 9-26.

Amigo, J., C. Ramírez \& L. Quintanilla 2004. The Nothofagus nitida (Phil.) Krasser woodlands of southern Chile in the northern half of their range: phytosociological position. Acta Botanica Gallica 151: 3-31.

Bjerke, J 2005. Synopsis of the lichen genus Menegazzia (Parmeliaceae, Ascomycota) in South America. Mycotaxon 91: 423-454.

Bjerke, J., A. Elvebakk \& W. Quilhot 2003. Distribución y ecología de las especies sorediosas de Menegazzia (Parmeliaceae, Ascomycota liquenizado) en Chile. Revista Chilena de Historia Natural 76: 79-98. 
Brunialti, G. \& P. Giordani 2003. Variability of lichen diversity in a climatically heterogenuos area (Liguria, NW Italy). Lichenologist 35: 55-69.

Calvelo, S. \& S. Liberatore 2004. Aplicability in situ of transplanted lichens for assessment of atmospheric pollution in Patagonia, Argentina. Journal of Atmospheric Chemistry 49: 199-210.

Di Castri, F. \& E. Hajek 1975. Bioclimatología de Chile. Ediciones Universidad Católica de Chile, Santiago. 128 pp.

Frey, W. \& R. Lösch 1998. Lehrbuch der Geobotanik - Pflanze und Vegetation in raum und Zeit. Gustav Fischer, Stuttgart. 436 pp.

Galloway, D. J. 1985. Flora of New Zealand Lichens. New Zealand Government Printer, Wellington. $662 \mathrm{pp}$.

Galloway, D. J. 1986. Non-glabrous species of Pseudocyphellaria from southern South America. Lichenologist, 18: 105-168.

Galloway, D. J. 1992a. Studies in Pseudocyphellaria (lichens) III. The South American Species. Biblioteca Lichenologica, 275 pp.

Galloway, D. J. 1992b. Lichens of Laguna San Rafael, Parque Nacional "Laguna San Rafael", southern Chile: indicators of environmental change. Global Ecology and Biogeography Letters 2: 37-45.

Galloway, D. J. 1994. Studies on the lichen genus Sticta (Schreber) Ach: I. Southern south american species. Lichenologist 26: 223-282.

Galloway, D. J. 1996. Lichen biogeography. En: Ed: Nash III, Th. Lichen Biology. Arizona State University. Cambridge University Press. 315-335.

Grube, M. \& D. L. Hawksworth 2007. Trouble with lichen: the re-evaluation and re-interpretation of thallus form and fruit body types in the molecular era. Mycological Research 111: 1116-1132.

Hajek, E. \& F. Di Castri 1975. Bioclimatografía de Chile. Ediciones Universidad Católica de Chile, Santiago. 114 pp.

Kuntze, H., G. Roeschmann \& G. Schwerdtfeger 1994. Bodenkunde. Eugen Ulmer, Stuttgart. $424 \mathrm{pp}$.

Lamb, M. 1958. Vegetación Liquénica de los Parques Nacionales Patagónicos. En: Anales de los Parques Nacionales, Buenos Aires. $188 \mathrm{pp}$.

Larcher, W. 1994. Ökophysiologie der Pflanzen. UTM für Wissenschaft Eugen Ulmer, Stuttgart. 394 pp.

McCune. B. \& L. Geiser 1997. Macrolichens of the Pacific Northwest. Corvallis, OR: Oregon State University Press. 386 pp.

Novoa, R. \& S. Villaseca 1989. Mapa agroclimático de Chile. Instituto de Investigaciones Agropecuarias (INIA) Santiago. $221 \mathrm{pp}$.

Oberdorfer, E. 1960. Planzensoziologische Studien in Chile - Ein Vergleich mit Europa. Flora et vegetatio Mundi 2: 1-208.

Pereira, I. 2007. Micobiota liquenizada del Parque Katalapi, X Región, Chile. Gayana Botánica 64: 192-200.

Pereira, I. \& J. San Martin 1998. Flora liquénica cortícola en un bosque caducifolio de Nothofagus alessandri, de Chile central. Cryptogamie Bryologie Lichenologie 19: 59-72.

Pereira, I., J. San Martín \& C. Roux 1999. Patrón florístico de líquenes epífitos de Nothofagus glauca en un bosque costero de Chile central. Gayana Botánica 56:69-76.

Pisano, E. 1981. Bosquejo fitogeográfico de FuegoPatagonia. Anales Instituto Patagonia (Chile) 12:159-171.

Pisano, E. 1983. The magellanic tundra complex. En: AJP. Gore (ed.) Mires, swamp, bog, fen and moor. Elsevier Scientific Pub. Company. Amsterdam. 295-329.

Pharo, E. \& A. Beattie 2002. The association between substrate variability and bryophyte and lichen diversity in Eastern Australian forest. The Bryologist 105:11-26.

Ramírez, C. 1968. Die Vegetation der Moore der Cordillera Pelada, Chile. Bericht der Oberhessisch. Ges. für Natur- und Heilkunde zu Giessen NF. Naturwiss. Abt. 36: 95-101.

Ramírez, C. \& V. Finot 2006. Ruderal flora from the city of Valdivia (Chile) and their ecological indicator-value spectra. Revista Geográfica de Valparaíso 38: 69-80.

Ramírez, C., C. San Martin \& H. Rubilar 2002. Una propuesta para la clasificación de los humedales chilenos. Revista Geográfica de Valparaíso 33: 265-273. 
Redon, J., L. Arellano \& M. Riveros 1979. Los líquenes de la Cordillera Pelada I. Estudio preliminar. Medio Ambiente 4: 71-79.

Sáiz, F. 1980. Experiencias en el uso de criterios de similitud en el estudio de comunidades. Archivos de biología y medicina experimental 13: 387-402.

San Martín, C., C. Ramírez \& M. Álvarez 2004. Estudio de la vegetación de "Mallines" y "Campañas" en la Cordillera Pelada (Valdivia, Chile). Revista Geográfica de Valparaíso 35: 261-273.

San Martín, C., C. Ramírez \& H. Figueroa 1999. Análisis multivariable de la vegetación de un complejo de turberas en Cordillera Pelada (Valdivia, Chile). Lazaroa 20: 95-106.

Scherhag, R. 1969. Klimatologie. Georg Westermann Verlag. Braunschweig. 165 pp.

Stenross, S. 1995. Cladoniaceae (Lecanorales, Ascomycotina Liquenizada), en la flora de Chile. Gayana Botánica 52: 89-131.
Teneb, E. \& O. Dollenz 2004. Distribución espacial de la flora vascular, la humedad y el pH en un turbal de esfagno (Sphagnum magellanicum Brid), Magallanes, Chile. Anales Instituto Patagonia (Chile) 32:5-12.

Valenzuela, J. \& R. Schlatter 2004. Las turberas de Cordillera Pelada provincia de Valdivia (X ${ }^{\mathrm{a}}$ Región, Chile). En: D. Blanco \& V. de la Balze (eds.) Los turbales de la Patagonia: Bases para su inventario y conservación de su biodiversidad. Publicación $\mathrm{N}^{\circ} 19$ : 87-92. Wetlands Internacional-America del Sur, Buenos Aires, Argentina.

Wedin, M. 1995. The lichen family Sphaerophoraceae (Caliciales, Ascomycotina) in temperate areas of the Southern hemisphere. Acta Universitattis Upsaliensis, Symbolae Botanicae Upsalienses 31:1.

Wilmanns, O. 1989. Ökologische Pflanzensoziologie. Quelle \& Meyer, Heidelberg. 378 pp. 